\title{
A Verification of Treatment Protocol in Fractionated Radiotherapy by Biological Dosimetry
}

\author{
İdil ÖNER ${ }^{1 *}$, Tuncay ORTA ${ }^{1}$, Melek Özlem KOLUSAYIN², Süreyya GÜNEBAKAN ${ }^{1}$, Asuman Kaynar Öztaş ${ }^{3}$ and Mustafa Ünsal ${ }^{3}$
}

${ }^{1}$ Faculty of Science, Department of Biology, Istanbul, Istanbul University, Turkey

${ }^{2}$ Cerrahpasa Medical Faculty Forensic Medicine Department, Istanbul University, Istanbul, Turkey

${ }^{3}$ Okmeydanı Educational and Researh Hospital, Radiation Oncology, Istanbul University, Istanbul, Turkey

\begin{abstract}
In radiation therapy of cancer, any possible mistakes that may occur in prescribed tumour dose calculation and/ or in treatment set up could also be checked by the biological dosimetry. In order to verify this, 20 patients having pelvic area tumours with no prescribed chemotherapy were selected. Their peripheral blood samples were collected before starting fractionated radiotherapy and at some definite time during treatment. Equivalent whole body doses (EWBD) were calculated from patient's weights, irradiated volumes and total tumor doses given until the date of blood sample collection during treatment. Each patient's EWBD was given in vitro to blood samples that were collected before starting radiotherapy. Radiation induced chromosome damage in lymphocytes were measured by micronucleus (MN) induction.

The relationship between the micronucleus frequencies (MN/BN) scored during therapy (in vivo) and the micronucleus frequencies scored after the application of in vitro EWBD was investigated. MN/BN rates scored following in vitro application of EWBD differed between 0.195 and 0.540 , whereas MN/BN rates obtained during radiotherapy equivalent to EWBD differed between 0.213 and 0.625 . It was shown that there was no significant difference $(p=0.653)$ between the MN scores following in vitro applied EWBD and MN scores obtained in vivo. The existence of this correlation has confirmed the correct application of fractionated radiotherapy and treatment setup for the patients.
\end{abstract}

Keywords: Biological Dosimetry; Radiotherapy; Micronucleus; Lymphocytes; Pelvic Tumors

\section{Introduction}

Use of radiation therapy in cancer treatment is one of the important treatment modalities. It can be used alone in treating many local cancers leading to a long time tumor control with good cosmetic results [1]. It may also be used in combination with surgery and/or chemotherapy. Radiotherapy standarts should be raised in order to provide a better tumor control. In addition to requirements in scientific research for raising standarts, radiation dose calculation and preparing the treatment setup for patient according to the treatment protocol is important in delivering accurate prescribed fractional radiation dose to tumor site [2]. For this reason, control of the treatment setup during the treatment period should be ensured by the physician, radiation physicist and radiotherapy technician in collaboration with each other.

Understanding any possible mistake at radiation dose calculations and/or treatment setup could also be possible by biological dosimetry which is based on measuring radiation induced chromosome damage [3]. In biological dosimetry, absorbed radiation dose estimations are generally made by scoring dicentric chromosome aberrations $[4,5,6,7]$. Background frequencies of this kind of unstable chromosome aberrations in healthy people with no radiation working history are very low [8]. Therefore dicentric formation is mostly related to radiation induction and there is a relationship between dicentric aberration frequency and radiation dose $[5,6,9]$. Scoring dicentric chromosomes is diffficult and takes a long time. A necessity arises in some applications of biological dosimetry in order to get results in a shorter time. Measuring micronuclei (MN) in binucleate cells is one of the techniques which meet with this requirement.

Micronucleus may represent acentric chromosome fragment or a whole chromosome that may be lost during segregation [10]. Micronuclei formation in human peripheral lymphocytes has been shown by the investigators to be a good representative of radiation damage $[10,11,12,13]$. In this study, $\mathrm{MN}$ technique was applied to peripheral blood lymphocytes of the radiotherapy patients having pelvic site tumors in order to verify the correct delivery of the prescribed tumor dose and treatment set-up during their fractionated treatment.

\section{Materials and Methods}

\section{Patient selection}

20 patients with pelvic site tumors were choosen for this study. Their ages varied between 35 and 82 . They had either earlier stage of disease or no chemotherapy following surgery. Patient's ages, tumor sites, tumor stages and treatment protocols were given in Table 1. Patients were told that their peripheral blood were taken before they start and during a definite time of radiotherapy in order to verify the prescribed tumor dose and treatment set-up. Patient's weights were measured before they start to radiotherapy for calculating their integral doses and equivalent wole body doses. They were treated by either 18 MV X-rays (Linear accelerator, Linac, PHILIPS) or gamma rays (Co-60 THR 780 E or Co-60 ATC).

${ }^{*}$ Corresponding author: İdil Öner, M.Sc, Istanbul University, Faculty of Science Department of Biology, Vezneciler, 34459, Istanbul, Turkey, E-mail: idiloner@ hotmail.com

Received July 19, 2011; Accepted July 30, 2011; Published July 31, 2011

Citation: ÖNER İ, ORTA T, KOLUSAYIN MÖ, GÜNEBAKAN S, Öztaş AK, et al. (2011) A Verification of Treatment Protocol in Fractionated Radiotherapy by Biological Dosimetry. J Nucl Med Radiat Ther 2:116. doi:10.4172/21559619.1000116

Copyright: ( 2011 ÖNER I, et al. This is an open-access article distributed under the terms of the Creative Commons Attribution License, which permits unrestricted use, distribution, and reproduction in any medium, provided the original author and source are credited. 
Citation: ÖNER I, ORTA T, KOLUSAYIN MÖ, GÜNEBAKAN S, Öztaş AK, et al. (2011) A Verification of Treatment Protocol in Fractionated Radiotherapy by Biological Dosimetry. J Nucl Med Radiat Ther 2:116. doi:10.4172/2155-9619.1000116

Page 2 of 5

\section{Integral dose and equivalent whole body dose calculations}

Integral doses were calculated according to the John's formulation [14]; $\Sigma=1.44 \times \mathrm{x}_{0} \times \mathrm{A} \mathrm{x} \mathrm{d}_{1 / 2} \mathrm{x}\left(1-\exp \left(-0.6938 \mathrm{~d} / \mathrm{d}_{1 / 2}\right) \mathrm{x}\left(1+2.88 \mathrm{~d}_{1 / 2} / \mathrm{f}\right)\right.$ / 1000 where, $D_{0}=$ given radiation dose, $A=$ irradiated area, $d_{1 / 2}=50 \%$ depth dose of isodose curves, $\mathrm{f}=$ source skin distance (SSD) and $\mathrm{d}=$ patient thickness.

Equivalent whole body doses (EWBD) for each patient were calculated dividing integral doses by patient's weights before they start to therapy. EWBD defines the total radiation dose that body acquires during treatment [9].

\section{Blood sampling}

Peripheral blood samples were taken into $5 \mathrm{ml}$ heparinized tubes before start and during between 8-12 days of their treatment.

\section{In vitro radiation application}

Equivalent whole body doses (EWBD) that were calculated for each patient from their body weights, irradiated volumes and tumor doses were given in vitro. EWBD's were kept to be between 2-3 Gy to compare in vitro and in vivo MN frequencies [15]. In vitro irradiation of any particular patient's blood was performed by the radiotherapy unit that was used to threat that particular patient. Blood tubes were irradiated at high dose rate inside the bolus material.

\section{Lymphocyte culture}

Microculture method [16] was applied for blood culture with minor modifications. $0.5 \mathrm{ml}$ of $5 \mathrm{ml}$ blood that was either irradiated in vitro or taken in vivo was put into culture containing phytohemaglutinin (PHA, $15 \mu \mathrm{g} / \mathrm{ml}$ ), $4 \mathrm{ml}$ of RPMI-1640 culture medium (Sigma, USA) and $1 \mathrm{ml}$ of Newborne Calf Serum. Cultures were incubated at $37^{\circ} \mathrm{C}$ for 45 hours. Cytochalasin-B (Sigma, USA) at $6 \mu \mathrm{g} / \mathrm{ml}$ was added $[17,13]$ to inhibit cell division and cultures were left another 24 hours in the incubator. At the end of 69 hours of total incubation period, cultures were treated by hypotonic KCI solution (0.075) and then cenrifuged for 3 times by methanol: acetic acid solution at 7:1 (v:v) ratio. Fixed cells were dropped onto glass slides and then stained by $5 \%$ Giemsa solution.

\section{Cell counting}

Cells having two identical nuclei were considered as binucleate cell under the light microscope with 400 magnifications. Nuclei like formations outside the main nuclei and no bigger than half of any daughter nucleus have been scored as micronucleus. Binucleate cells with or without $\mathrm{MN}$ were scored and $\mathrm{MN}$ frequencies for each patient were calculated.

\section{Statistics}

The relationship between in vivo $\mathrm{MN}$ frequencies obtained during treatment and MN frequencies obtained after application of EWBD in vitro was evaluated by Spearman correlation test [18]. Correlation between in vivo and in vitro $\mathrm{MN}$ frequencies was evaluated by the paired t-test [18].

\section{Results}

\section{Patient's treatment and dosimetry}

All the patients received 50 Gy radiation dose in 25 fractions as being 2 Gy/fraction (Table 1). Irradiated volume and area on patient's skin, body weights, and planned radiation dose given to tumor by the time blood samples were taken during treatment, integral dose and EWBD of patients by that time were given in Table 2. Blood that were taken before patients start to radiotherapy were given in vitro radiation dose equal to calculated whole body dose of fractionated treatment at the time of blood collection. For this, body weights of patients before entering therapy were measured and treatment area of patients was calculated.

Integral doses were calculated after adding total skin dose embarked until blood collection during treatment. EWBD for each patient was calculated as dividing integral dose by body weight.

\begin{tabular}{|c|c|c|c|c|c|}
\hline Patient & Age (years) & Tumor type and stage & Radiation source & Fraction number & Total dose (Gy) \\
\hline 1 & 47 & vagina ca. stage; IIB & Co-60 THR & 25 & 50 \\
\hline 2 & 51 & Op. corpus ca. stage; IC & Co-60 THR & 25 & 50 \\
\hline 3 & 49 & Op. cervix ca. stage; IC & Co-60 THR & 25 & 50 \\
\hline 4 & 74 & Inop. cervix ca. stage; IIB & Co-60 THR & 25 & 50 \\
\hline 5 & 74 & Op. endo. ca. stage; IIA & Co-60 THR & 25 & 50 \\
\hline 6 & 64 & Op. endo. ca. stage; IC & Linac-18 MV & 25 & 50 \\
\hline 7 & 43 & Cervix ca stage; IIB & Co-60 THR & 25 & 50 \\
\hline 8 & 56 & Op. endo. ca. stage; IIIA & Co-60 THR & 25 & 50 \\
\hline 9 & 58 & Op. endo. ca. stage; IIB & Co-60 THR & 25 & 50 \\
\hline 10 & 35 & Op. endo. ca. stage; IIB & Co-60 THR & 25 & 50 \\
\hline 11 & 73 & Op. endo. ca. stage; IC & Co-60 THR & 25 & 50 \\
\hline 12 & 45 & recur. over ca. stage; recur & Co-60 THR & 25 & 50 \\
\hline 13 & 71 & Inop. cervix ca. stage; IIB & Co-60 THR & 25 & 50 \\
\hline 14 & 54 & Endo. ca. stage; IIB & Co-60 ATC & 25 & 50 \\
\hline 15 & 55 & Op. endo. ca. stage; IIIA & Co-60 THR & 25 & 50 \\
\hline 16 & 69 & Op. cervix ca. stage; IB & Co-60 THR & 25 & 50 \\
\hline 17 & 43 & endo. ca. stage; IB & Co-60 ATC & 25 & 50 \\
\hline 18 & 54 & Op. endo. ca. stage; IIA & Co-60 ATC & 25 & 50 \\
\hline 19 & 82 & Op. endo. ca. stage; IC & Co-60 THR & 25 & 50 \\
\hline 20 & 49 & Op. endo. ca.stage; IC & Linac 18-MV & 25 & 50 \\
\hline
\end{tabular}

ca; carcinoma, Op; operated, Inop; inoperated, endo; endometrium, recur; recurrent

Table 1: Patients ages, tumor types and treatment protocols. 
Citation: ÖNER I, ORTA T, KOLUSAYIN MÖ, GÜNEBAKAN S, Öztaş AK, et al. (2011) A Verification of Treatment Protocol in Fractionated Radiotherapy by Biological Dosimetry. J Nucl Med Radiat Ther 2:116. doi:10.4172/2155-9619.1000116

Page 3 of 5

\begin{tabular}{|c|c|c|c|c|c|c|c|}
\hline Patient & Irradiated volume $\left(\mathrm{cm}^{3}\right)$ & Irradiated area $\left(\mathrm{cm}^{2}\right)$ & Body weight (kg) & Fraction number & Tumor dose (Gy) & Integral dose (kg.Gy) & EWBD (Gy) \\
\hline 1 & 7344 & 306 & 86 & 10 & 20 & 227.55 & 2.65 \\
\hline 2 & 6072 & 264 & 66 & 10 & 20 & 186.18 & 2.82 \\
\hline 3 & 4050 & 225 & 49 & 11 & 22 & 130.35 & 2.66 \\
\hline 4 & 4950 & 247.5 & 59 & 10 & 20 & 146.89 & 2.49 \\
\hline 5 & 4374 & 182.3 & 60.5 & 11 & 22 & 149.29 & 2.47 \\
\hline 6 & 6075 & 225 & 94 & 10 & 20 & 190.63 & 2.03 \\
\hline 7 & 4862 & 221 & 64.5 & 9 & 18 & 134.73 & 2.09 \\
\hline 8 & 5760 & 288 & 66 & 11 & 22 & 190.52 & 2.89 \\
\hline 9 & 5460 & 227.5 & 58 & 9 & 18 & 156.13 & 2.69 \\
\hline 10 & 5184 & 288 & 66 & 11 & 22 & 167.66 & 2.54 \\
\hline 11 & 4116 & 196 & 57 & 12 & 24 & 160.24 & 2.81 \\
\hline 12 & 6992 & 304 & 67 & 9 & 18 & 194.00 & 2.90 \\
\hline 13 & 7200 & 300 & 71.5 & 9 & 18 & 201.07 & 2.81 \\
\hline 14 & 6336 & 264 & 97 & 11 & 22 & 216.28 & 2.23 \\
\hline 15 & 7812 & 279 & 83.5 & 10 & 20 & 253.82 & 3.03 \\
\hline 16 & 6090 & 203 & 99 & 11 & 22 & 223.86 & 2.26 \\
\hline 17 & 5003 & 217.5 & 57.5 & 11 & 22 & 168.69 & 2.93 \\
\hline 18 & 4774 & 217 & 70 & 11 & 22 & 159.73 & 2.28 \\
\hline 19 & 6448 & 248 & 93 & 11 & 22 & 224.81 & 2.42 \\
\hline 20 & 4950 & 225 & 67 & 12 & 24 & 180.84 & 2.70 \\
\hline
\end{tabular}

Table 2: Physical measures and dose calculations of patients.

\begin{tabular}{|c|c|c|c|c|c|c|c|}
\hline \multirow{2}{*}{ Patient } & \multirow{2}{*}{ EWBD (Gy) } & \multicolumn{3}{|c|}{ Scores obtained after EWBD application in vitro before treatment } & \multicolumn{3}{|c|}{ Scores obtained after in vivo EWBD during therapy } \\
\hline & & BN cell & MN & MN/BN & BN cell & MN & MN/BN \\
\hline 1 & 2.65 & 363 & 116 & 0.319 & 187 & 69 & 0.368 \\
\hline 2 & 2.82 & 403 & 86 & 0.213 & 1487 & 365 & 0.245 \\
\hline 3 & 2.66 & 1113 & 602 & 0.540 & 296 & 185 & 0.625 \\
\hline 4 & 2.49 & 934 & 330 & 0.353 & 723 & 247 & 0.342 \\
\hline 5 & 2.47 & 708 & 187 & 0.264 & 523 & 135 & 0.258 \\
\hline 6 & 2.03 & 215 & 73 & 0.339 & 339 & 106 & 0.312 \\
\hline 7 & 2.09 & 218 & 70 & 0.321 & 950 & 310 & 0.326 \\
\hline 8 & 2.89 & 375 & 148 & 0.379 & 744 & 252 & 0.339 \\
\hline 9 & 2.69 & 394 & 110 & 0.279 & 214 & 60 & 0.280 \\
\hline 10 & 2.54 & 168 & 39 & 0.232 & 546 & 128 & 0.234 \\
\hline 11 & 2.81 & 418 & 114 & 0.273 & 289 & 77 & 0.266 \\
\hline 12 & 2.90 & 485 & 134 & 0.276 & 425 & 101 & 0.238 \\
\hline 13 & 2.81 & 331 & 67 & 0.202 & 220 & 50 & 0.227 \\
\hline 14 & 2.23 & 221 & 43 & 0.195 & 582 & 118 & 0.272 \\
\hline 15 & 3.03 & 353 & 77 & 0.201 & 624 & 150 & 0.240 \\
\hline 16 & 2.26 & 478 & 125 & 0.262 & 610 & 130 & 0.213 \\
\hline 17 & 2.93 & 387 & 86 & 0.222 & 527 & 126 & 0.239 \\
\hline 18 & 2.28 & 576 & 129 & 0.224 & 504 & 126 & 0.25 \\
\hline 19 & 2.42 & 693 & 150 & 0.216 & 632 & 142 & 0.225 \\
\hline 20 & 2.70 & 225 & 60 & 0.267 & 194 & 48 & 0.247 \\
\hline
\end{tabular}

Table 3: MN frequencies before and during therapy.

Micronucleus frequencies obtained in vitro and during radiotherapy

Number of binucleate cells and number of micronuclei in these cells at different tumor doses before and during radiotherapy were given in Table 3. $\mathrm{MN}$ frequencies $(\mathrm{MN} / \mathrm{BN})$ were the ratio of micronuclei to binucleate cells.

$\mathrm{MN} / \mathrm{BN}$ ratios after application of in vitro EWBD were between 0.195 and 0.540 and MN/BN ratios after the embarkment of the same EWBD during radiotherapy were between 0.213 and 0.625 (Figure 1). Analyzing these data by the Spearman correlation test showed an existence of the significant relationship $(\mathrm{r}=0.847)$ between $\mathrm{MN}$ frequencies obtained after in vitro equivalent dose and $\mathrm{MN}$ frequencies obtained fractionated therapy (in vivo). \% 95 significance levels of the correlation coefficient were between 0.638 and 0.940 . Application of paired t-test to data indicated that there was no statistical difference ( $\mathrm{p}=0.653$ ) between the in vitro and in vivo $\mathrm{MN}$ frequencies as shown below.

\section{Results of paired t-test}

$\mathrm{X}$ (average) $\pm \mathrm{SD}$ (standart deviation) (n)

In vitro values

$0.281 \pm 0.082(20)$

In vivo values

$0.284 \pm 0.093(20)$

Average difference

$0.003 \pm 0.011(20)$

Statistical significance

$\mathrm{t}=0.457: \mathrm{p}=0.653$ 


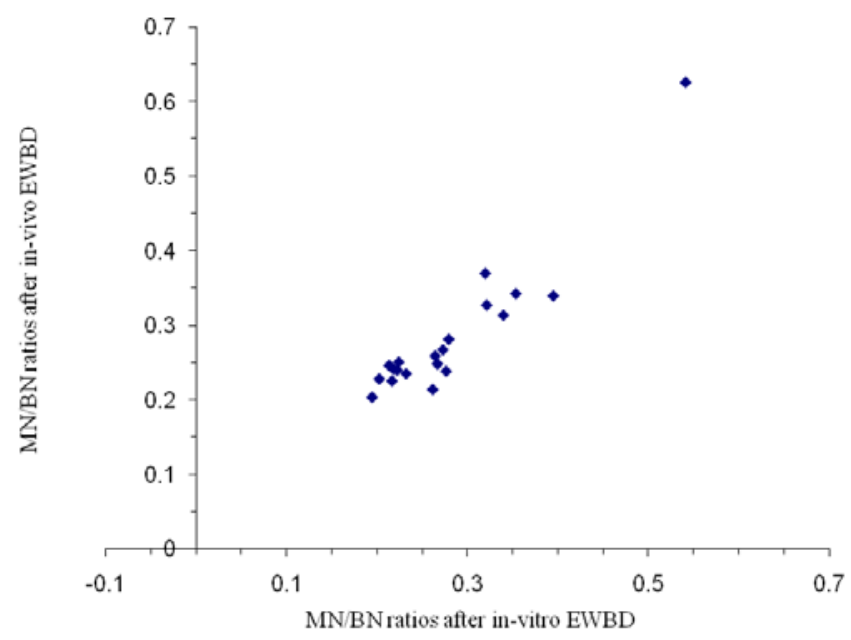

Figure 1: The relationship between the MN/BN ratios obtained in vitro and in vivo EWBD.

\section{Discussion}

The aim of radiotherapy is to deliver an optimal radiation dose to tumor depending on dose tolerance levels of normal tissue. Radiotherapy plays an important role in the treatment of pelvic malignancies as being a primer treatment or adjuvant treatment combined with surgery [19,20]. Effective radiation doses can be delivered to treatment areas including all pelvis. There is a rising interest in recent years towards using high doses of radiation to control tumor and to increase treatment ratios. However radiotherapy may also lead to permanent and/or temporary damage in normal tissue $[21,22]$. The main side effects are gastrointestinal and genitouriner symptoms [22].

Two techniques may be used in planning radiation treatment fields. One of them is the multiple field technique which reduces the amount of small intestine from treatment volume. The other technique which was used in this study is called AP/PA (anteroposterior/posteroanterior) field technique. This technique allows more amount of small intestine to be included in treatment volume and therefore total radiation dose is generally limited to 45-50 Gy. The aim of conventional high energy radiotherapy techniques is to hold tumor integral dose lower compared to general integral dose of patient [23]. In other words, a favorable choice in patient treatment planning is to obtain the smallest integral dose for tumor.

Lower integral dose ratios are obtained when $10 \mathrm{MV}$ or over radiation energies are choosen for treatment of deeply located and big sized lesions in pelvis/abdominal area [24]. When \% dose levels of deeply located tumors are decreased with increasing depth, smaller integral doses which are appropriate for tumor treatment planning may be obtained. Integral doses were calculated by John's formulation [14] in this study where Cobalt- 60 gamma rays and high energy photons were used for patient's treatment. Patient's integral doses have been observed to increase with an increasing irradiated tumor volumes in this study (Table 2). Integral dose of one of the two patients (numbered 8 and 10 with) with larger volume was higher although their irradiated area and fraction number were the same. When the comparision was made between the results of Co-60 teletherapy machines (ATC-C9 and THR $780 \mathrm{E}$ ) and Lineer accelerator irradiations, integral doses of patients treated by by ATC-C 9 were also higher. These results might be due to technical differences of treatment machines. Not significant differences in integral doses were observed when the comparision was made between $2^{\text {nd }}$ numbered patient treated by Co-60 THR $780 \mathrm{E}$ and $6^{\text {th }}$ numbered patient treated by lineer accelerator.

Tumor dose should correctly be calculated, patient treatment setup that is planned for patient should appropriately prepared before patient starts to therapy and shoul also be kept unchanged during patients treatment. These activities should always be checked by the physician, physicist and technician. Any possible mistake that might occur during dose calculations and/or treatment setup may result in under or over exposure of patient. Understanding this mistake may also be possible with biological dosimetry which is based on measuring radiation induced chromosome damage. Cytokinesis-blocked micronucleus method (CBMN) provides fast and economical way of measuring chromosome damage in human lymphocytes [10]. Micronucleus can be acentric chromosome fragments or a whole chromosome that can be lost during segregetion [10]. There have been many studies showing the utility of the technique in biological dosimetry [3,10,12,16,17,25]. Studies that were performed with radiotherapy patients have also confirmed that MN technique is reliable and conveinent technique in estimating equivalent whole body dose that patient receives during fractional treatment $[9,11,26,27,28]$

In this study, in order to check the accuracy of tumor dose calculations and treatment setups biologically, micronuclei in binucleate cells of peripheral lymphocytes taken before starting and during fractional radiotherapy applied to pelvic area tumors of 20 patients were scored. MN frequencies have been observed to increase with an increasing radiation dose in radiation dose response studies $[15,29]$, but ratio of increases declined at 4-5 Gy of radiation doses. Encasing aberrations at high doses by any of sister nuclei or micronuclei might be responsible from this decline. In this study, therefore, equivalent whole body radiation doses were kept between 2 and $3 \mathrm{~Gy}$. There was a significant relationship $(\mathrm{r}=0.847)$ between $\mathrm{MN} / \mathrm{BN}$ ratios obtained during fractional therapy and $\mathrm{MN} / \mathrm{BN}$ ratios obtained in vitro application of EWBD before start of radiotherapy (Figure 1). The evidence of significant relationship confirmed the planned radiation dose for patients had been delivered appropriately to tumor through lined treatment area on patients.

Interaction of radiation with matter in tumor irradiation is the same either in tumor cells or normal cells along radiation track, therefore ionising radiation does not discriminate neoplastic cells [22]. This interaction in normal tissue and cells is responsible from the onset of early and/or late normal tissue complications that might occur in radiotherapy. Eventually, eventhough transfered treatment dose to patient could be technically calculated, radiation damage occuring in lymphocytes might represent normal tissue damage. The existence of equivalancy in chromome damage measured during radiotherapy and after application of EWBD in vitro in this study showed that patients had received planned radiation dose to tumor. In addition, patient treatment setups were inspected carefully and therefore their accuracy were confirmed during this study. But for any other therapies to be correct in delivering fractionated dose to tumor, beside proper calculation of prescribed tumor dose, it is also equally important to check patient treatment setup during radiotherapy. Because radiation that was planned to give to lined area on patient by teletherapy machine might deflect outside lined area with the same treatment portal resulting in an excepted integral dose but under dosed tumor volume. Physical control of treatment setup should be ensured carefully by clinician, radiation physicist and radiotherapy technician and it is crucially important to maintain this original setup by tecnician during fractionated therapy. 
Citation: ÖNER I, ORTA T, KOLUSAYIN MÖ, GÜNEBAKAN S, Öztaş AK, et al. (2011) A Verification of Treatment Protocol in Fractionated Radiotherapy by Biological Dosimetry. J Nucl Med Radiat Ther 2:116. doi:10.4172/2155-9619.1000116

Page 5 of 5

This study which was performed with pelvic area tumors with different tumor stages and different irradiated areas can also be performed with other area tumors to check whether treatment protocols are performed correctly.

\section{Acknowledgements}

This work was supported by Istanbul University, Research Fund (Project no: T-70/23072002).

\section{References}

1. Steel GG (1992) The significance of radiobiology for radiotherapy. In Steel GG Ed The ESTRO Book of Basic Clinical Radiobiology ESTRO Teaching Course, Aarhus, Denmark: 1-6.

2. Meredith WJ, Massey JB (1968) Patient dosage. In Fundamental physics of radiology, John Wright \& Sons Ltd, 423-427.

3. Zoetelief J, Broerse JJ (1990) Dosimetry for radiation accidents: present status and prospects for biological dosemeters. Int J Radiat Biol 57: 737-750.

4. Berek JS, Fu YS, Hacker NF (1996) Ovarian Cancer. In: Berek JS, Adashi EY, Hillard PA, eds. Novak's Gynecology 20th ed Baltimore, Maryland, Williams \& Wilkins 351 West Camden Street: 1155-1230.

5. Scheid W, Weber J, Traut H (1993) Chromosome aberrations induced in the lymphocytes of pilots and stewardnesses. Naturwissenschaften 80: 528-530.

6. Traut $\mathrm{H}$ (1990) Procedure for calculating the radiation induced chromosome aberrations after exposure during several years. Studia Biophysica 138: 201213.

7. Weber J, Scheid W, Traut H (1995) Biologic dosimetry after extensive diagnostic Xray exposure. Health Physics 68: 266-269.

8. Bauchinger M (1995) Quantification of low-level radiation exposure by conventional chromosome aberration analysis. Mutat Res 339: 117-189.

9. Venkatachalam P, Solomon FDP, Prabhu BK, Mohankumar MN, Gajendiran $\mathrm{N}$, et al. (1999) Estimation of dose in cancer patients treated with fractionated radiotherapy using translocation, dicentrics and micronuclei frequency in peripheral blood lymphocytes. Mutat Res 429: 1-12.

10. Fenech M (1997) The advantages and disadvantages of the cytokinesis-block micronucleus method. Mutat Res 392: 11-18.

11. Le Roux J, Slabbert J, Smit B, Blekkenhorst G (1998) Assesment of the micronucleus assay as a biological dosimeter using cytokinesis-blocked lymphocytes from cancer patients receiving fractioned partial body radiotherapy. Strahlenther Onkol 174: 75-81.

12. Lee TK, Wiley Jr AL, Means JA, Esinhart JD, Blackburn LD (1993) Frequencies of micronuclei in human lymphocytes: comparison between healthy donors and cancer patients and their in vitro response to ionizing radiation. Radiat Oncol Invest 1: 148-152.

13. Tunçel N (2002) Eksternal Radyoterapi Planlaması -1 (2D) UROK Temel Radyoterapi, Radyasyon Fiziği ve Radyobiyolojı Kurs Kitapçığı. 57-82.
14. Johns HE (1961) Introduction to Physics of Radiology. $2^{\text {nd }}$ ed 353-357.

15. Orta T, Günebakan S, Coşkun M, Bilge H (2001) Dose Response of Micronucle in Human Lymphocytes with Co-60 Gamma or X-Rays. The Scientific and Pedagogical News of Odlar Yurdu University 6: 134-142.

16. Moorhead PS, Novel PC, Mellmann WJ, Battips DM, Hungerford DA (1960) Chromosome preparations of leukocytes cultured from human peripheral blood. Exp Cell Res 20: 613-616.

17. Gantenburg HW, Wuttke K, Streffer C, Muller WU (1991) Micronuclei in human lymphocytes irradiated in vitro or in vivo. Radiat Res 128: 276-281.

18. Şenocak M (1990) Temel Bioistatistik. $1^{\text {st }}$ ed. Çağlayan Kitabevi, İstanbul.

19. Peters LJ, Ang KK (1998) Unconventional shemes in radiotherapy. Important adv Oncol 269-285.

20. Thomas GM, Dembo AJ, Beale F, Bean H, Bush R, Herman J, et al. (1984) Concurrent radiation, mitomycin $-\mathrm{C}$ and 5 -fluorouracil in poor prognosis carcinoma of the cervix: preliminary results of a phase I-II study. Int J Radio Oncol Biol Phys 10: 1785-1790.

21. Athanassiou H, Antonadou D, Coliarakis N, Kouveli A, Synodinou M (2003) Oncology Hellenic Group. Protective effect of amifostine during fractionated radiotherapy in patients with pelvic carcinomas: results of a randomized trial. Int J Rad Oncol Biol Phys 56: 1154-1160.

22. Young RC, Thomas GM General Principles of Cancer Therapy. In: Berek JS Adashi EY, Hillard PA, eds. Novak's Gynecology. 20th.ed. Baltimore, Maryland, Williams \& Wilkins 351 West Camden Street: 1996: 1015-1055

23. Verellen D, Vanhavere F (1999) Risk assessment of radiation-induced malignancies based on whole-body equivalent dose estimates for IMRT treatment in the head and neck region. Radiother Oncol 53: 199-203.

24. Pirzkall A, Carol M.P, Pickett B, Xia P, Roach III M (2002) The Effect of beam energy and number of field on photon-based IMRT for deep-seated targets. Int J Radiat Oncol Biol Phys 53: 434-442.

25. Müller WU, Nüsse M, Miller BM, Slavotinek A, Viaggi S (1996) Micronuclei: a biological indicator of radiation damage. Mutat Res 366: 163-169.

26. Catena C, Conti D, Parasacchi P, Marenco P, Bortolato B (1996) Micronucleis in cytokinesis blocked lymphocytes may predict patient response to radiotherapy. Int J Radiat Biol 70: 301-308.

27. Catena C, Parasacchi P, Conti D, Sgura A, Trenta G (1997) Peripheral blood lymphocyte decrease and micronucleus yields during radiotherapy. Int J Radiat Biol 72: 575-585.

28. Fenech M, Denham J, Francis W, Morley A (1990) Micronuclei in cytokinesisblocked lymphocytes of cancer patients following fractioned partial-body radiotherapy. Int J Radiat Biol 57: 373-383.

29. Paul SF, Venkatachalam P, Jeevanram RK (1997) Analysis of radiation doseresponse curve obtained with cytokinesis block micronucleus assay. Nucl Med Biol 24: 413-416. 